\title{
LiDAR Integrated High-Capacity IR OWC System with Abilities of Localization and Link Alignment
} \author{
AND H. Y. Fu' ${ }^{1,2, *}$ \\ ${ }^{1}$ Tsinghua Shenzhen International graduate school, Tsinghua University, Shenzhen 518055, China \\ ${ }^{2}$ Tsinghua-Berkeley Shenzhen Institute, Tsinghua University, Shenzhen 518055, China \\ *Corresponding author: hyfu@sz.tsinghua.edu.cn
}

ZHI LI ${ }^{1,2}$, YICONG LI ${ }^{1,2}$, ZIHAN ZANG ${ }^{1,2}$, MUTONG LI ${ }^{1,2}$, YAQI HAN ${ }^{1,2}$, LICAN WU ${ }^{1,2}$

Received XX Month XXXX; revised XX Month, XXXX; accepted XX Month XXXX; posted XX Month XXXX (Doc. ID XXXXX); published XX Month XXXX

By using narrow infrared (IR) beams, optical wireless communication (OWC) system can realize ultra-high capacity and high-privacy transmission. However, due to the point-to-point connection approach, a high-accuracy localization system and beam-steering antenna (BSA) are required to steer the signal beam to user terminals. In addition, to achieve link alignment in the receiver, the BSA needs to be within the limited receiver field of view (FoV). This problem greatly limits the practical application of high-capacity IR optical wireless communication and has not been well solved yet. In this paper, we proposed an indoor beam-steering IR OWC system with high-accuracy and calibration-free localization ability by employing a coaxial frequency modulated continuous wave (FMCW) light detection and ranging (LiDAR) system. In the meantime, benefit from the mm-level ranging accuracy of the LiDAR system, a feasible approach to deal with the link alignment issue for practical applications is firstly demonstrated. With the assistance of the LiDAR system, we experimentally achieved the localization of user terminals with a 0.038-degree localization accuracy, link alignment at the receiver and an error-free on-off keying $(0 \mathrm{OK})$ downlink transmission of $20 \mathrm{~Gb} / \mathrm{s}$ in free space at 3-m distance is demonstrated.

\section{Introduction}

Recently, the internet of things (IoT) network market has seen explosive growth [1]. As a result, the number of "things" (e.g. sensors and electronics devices), which require interconnection between each other and connection to the network, is increasing rapidly and a large volume of data traffic is generated. Thus, broadband data communication is highly demanded. However, nowadays widely applied radio frequency spectrum is getting overcrowded and exhausted [2,3]. Optical wireless communication (OWC) technique is a promising solution to solve this issue, which usually works at a higher frequency with a much more broad spectrum. Furthermore, OWC is also one of the potential candidates for $6 \mathrm{G}$ high-capacity transmission technique and has attracted tremendous attention [4, 5]. The visible light communication (VLC) system and infrared (IR) OWC system are the two most studied technologies for indoor OWC communication. VLC system utilizes LED as a transmitter, which can realize illumination and data transmission simultaneously. While IR OWC utilizes a narrow infrared laser beam as a carrier to transmit data signals. IR OWC system has the advantages of ultrahigh capacity, good privacy, eye safety, and high energy efficiency [6]. However, due to the narrow radiation angle of infrared laser beam, IR OWC system suffers from a few critical issues in practical indoor applications. The first issue is the small coverage area. Therefore, the beam-steering device is implemented to provide the system with a beam-steering field of view (FoV) to further improve the transmission coverage area [7-9]. Secondly, the ultrahigh transmission data rate can only be realized by the point-to-point connection approach. Therefore, the accurate localization of the receiver is needed. Many localization techniques have been

developed for IR OWC systems. Using an optical camera to realized localization and tracking is a feasible method, which can obtain high localization accuracy [10-12]. However, active LED ring is required as a marker to assist capture receiver and additional power consumption is inevitable. Besides, the camera and beam-steering device are not coaxial and system calibration is required. A fully passive localization approach is also proposed by using an optical corner retroreflector near the receiver, which is suitable for the beam with large diameter of the footprint [13], but the reflected beam suffers from a slight lateral offset. There are many other indoor position systems that have not been applied in IR OWC system, such as Zigbee-based indoor positioning and radio frequency identification(RFID) [14]. Another critical issue is the link alignment at the receiver.So far, high-speed infrared photodetectors have very limited active area with only around $30-\mu$ m diameter $[15$, 16]. Commercial products are usually packaged with fiber pigtails. The signal beam is required to be coupled into a multimode fiber or single-mode fiber. In the previously reported works, a collimator is widely used to receive narrow infrared signal beam. For practical applications, in addition to the accurate localization of the receiver, the beam-steering device must be within the FoV of the receiver so that the incident beam can be detected. Although many approaches have been reported to improve receivers' FoV, such as compound parabolic concentrator (CPC), hemispherical lens, and fiber optic bundle array [17-19]. However, a $180^{\circ}$ wide angle receiver is not available by now. Thus, to achieve link alignment in the receiver end, the beam-steering device must be placed within the receiver FoV. However, to the best of our knowledge, such issue has not been well discussed and solved yet in the previous reported works. But, in fact, 
(a)

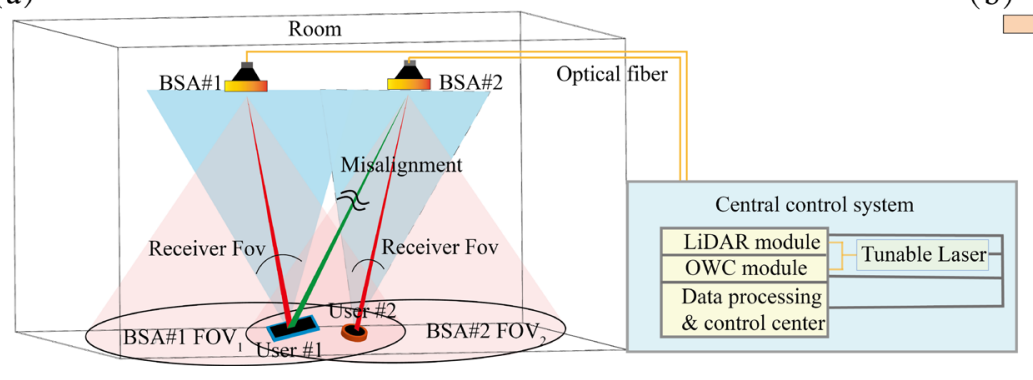

(b)

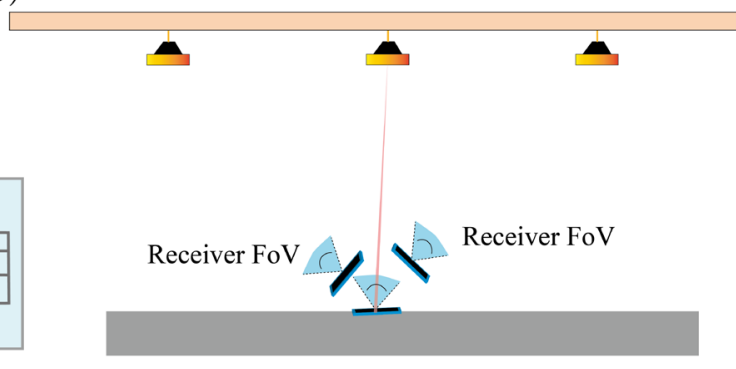

Fig. 1. (a)Schematic diagram of a LiDAR integrated infrared OWC system. (b) multiple BSAs is installed in a room. BSA: Beam-steering antenna

this problem greatly limits the practical application of high-capacity IR OWC system.

Recently, light detection and ranging (LiDAR) attracts lots of attention, which is widely used in autonomous vehicles, augmented reality (AR) and simultaneous localization and mapping (SLAM) [20-22]. As a commercial 3D sensor, LiDAR is promising to be implemented in indoor electronic devices. In this paper, we propose a high-capacity infrared beam-steering optical wireless communication system integrated with a coaxial frequency modulated continuous wave (FMCW) LiDAR system. The implemented LiDAR system can realize mm-level ranging accuracy, which is used for localization and tracking of user terminals. We suggest a simple circle object detection framework that incorporates Canny edge detection [23] and Hough transform system [24-26] techniques to demonstrate the localization ability of our system. The LiDAR system and the OWC system share a common beam-steering device, which means the system is calibration-free for localization and tracking. On the other hand, depth information from LiDAR system can be further used to measure the normal direction of the receiver surface, which offers an excellent solution to the link alignment issues discussed above. To the best of our knowledge, this is the first experimental demonstration of LiDAR applied in an infrared beam-steering OWC system, which can well address the issues of user localizationand link alignment in IR OWC system. We experimentally realize the localization of user terminal and an error-free on-off keying (OOK) transmission of $20 \mathrm{~Gb} / \mathrm{s}$ in free space at 3 meters is achieved. The proposed system can realize 0.038-degree localization accuracy. We also investigate the feasibility of the proposed system in measuring the tilt angle of the receiver surface. A spectral scanning method is employed for both infrared OWC system and LiDAR system. The advantage of such method is the inertia-free, fast and stable beam-steering. In addition, the 3D imaging pixel of the used LiDAR system is reconfigurable by sacrificing the ranging resolution and hence the localization accuracy is also adjustable.

\section{Architecture of LiDAR integrated infrared OWC system}

Figure 1(a) demonstrates the indoor beam-steering infrared OWC system with localization and link alignment abilities by using a coaxial LiDAR. Multiple beam-steering antennas (BSA) are installed at the top of the room which is fed by optical fibers from central control system. BSAs at the ceiling of the room can steer light beams to any position within its FoV. On the one hand, BSAs offer a large coverage area for narrow beam IR OWC system. On the other hand, BSAs also provides the LiDAR system with 2D beam-scanning ability. Here, a spectral scanning approach is adopted. 2D beamsteering can be achieved by diffractive element and wavelengthswept laser source. In our system, BSAs consist of a grating and a galvo scanning mirror[7, 27]. Before steering signal beams to the receiver aperture, the localization of user terminals is required for establishment of transmission link. Here, a FMCW LiDAR system with high ranging accuracy is used for objection detection and tracking. As depicted in Fig.1 (a), the OWC system and LiDAR system share the same BSA. Benefitting from such design, system calibration is notnecessary, which greatly reduces the complexity of system operation. Based on the 3D cloud points obtained from LiDAR module, the positions of user terminals can be captured in the data processing center. To improve the localization performance, a marker around the receiver aperture is installed, which is a card with high infrared reflectivity. Based on the extracted position, the corresponding output wavelength of laser source can be obtainedand then the signal beam can be steered into the receiver aperture by setting the wavelength of laser source. A programmable tunable laser in the central control system combined with BSA is utilized to realize such beam-steering function.

In the practical scene, only steering the narrow beam accurately to the center of the receiver's aperture is insufficient to achieve transmission link. Another issue is the establishment of link alignment. As illustrated in Fig. 1 (a), both BSA\#1 and BSA\#2 have their own respectively beam-steering FoVs. User \#1 is within their transmission coverage area. However, due to the limitation of receiver FoV of user \#1, only BSA \#1 can achieve link alignment with user \#1. Thus, a strict condition should be satisfied, which is that BSA should be within the receiver's FoV. However, in the practical mobile user communication scenario, the position and the placement angle of the receiver are random. Besides, even with the introduction of a wide-angle lens, it is still difficult for the receiver to achieve 180-degree FoV. To reduce the blind area of connection, multiple BSAs are installed in a room. There would always be a suitable BSA that is within the receiver's FoV and can achieve link alignment. The case is demonstrated in Fig. 1(b). By using the 3D cloud points of receiver surface, both the position and the tilt angle of receiver reference to the BSA can be accurately obtained. Based on the tilt angle of the receiver surface, the BSA which is within the FoV of the receiver can be selected and then link alignment can be realized.

In the followed section, we mainly discuss the achievement of 


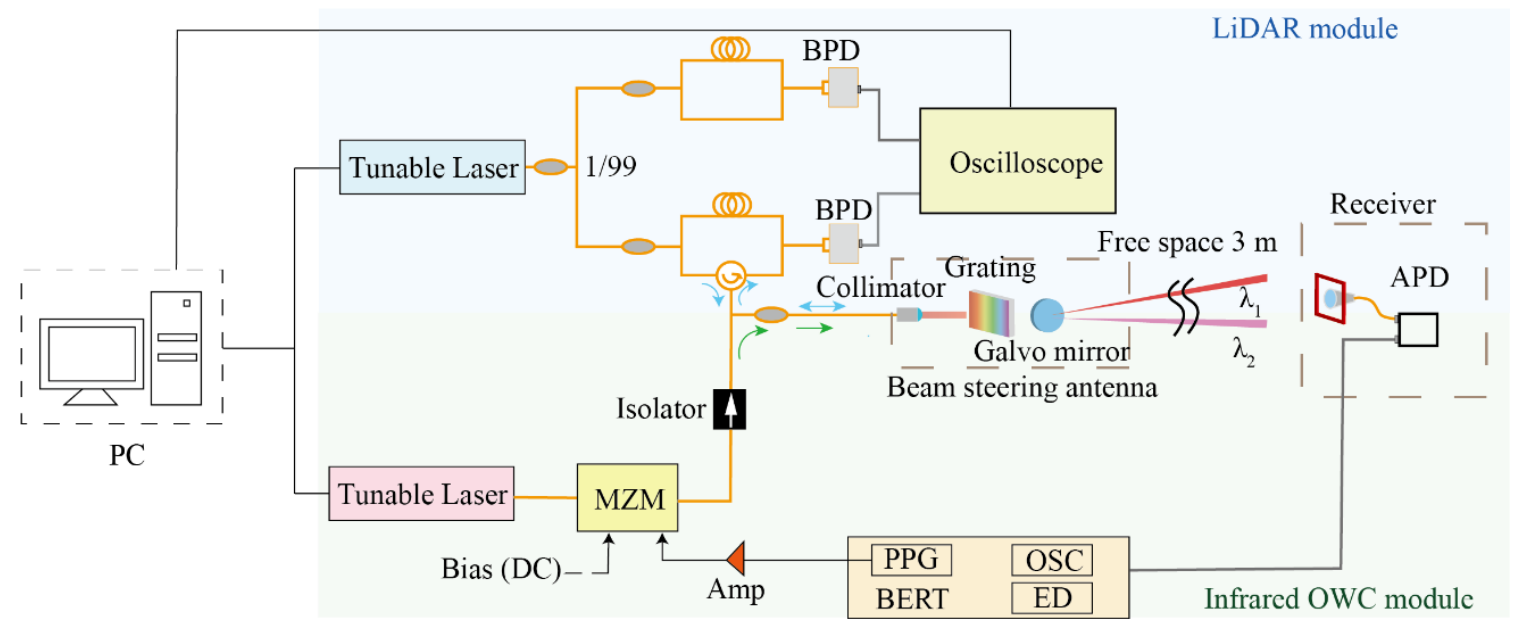

Fig. 2. Experiment setup for LiDAR integrated infrared OWC system. PC: Personal Computer; MZM: Mach-Zehnder modulator; BPD: Balanced Photodetector; PPG: pulse pattern generator; OSC: Oscilloscope; ED: Error Detector.

downlink transmission based on the localization and tilt angle measurement of receiver by using LiDAR system. High-capacity data transmission experiment results are also described.

\section{Experimental setup and results}

\subsection{LiDAR integrated OWC system}

Figure.2 demonstrates the experimental setup of LiDAR integrated OWC system. The system can be divided into two modules: LiDAR module and OWC module. The shared beam-steering antenna is composed of a grating and a Galvo scanner, which can provide 2D beam-steering ability combining with a tunable laser. A blazed grating (T-940L, Lightsmyth) with line density of 940.7 line $/ \mathrm{mm}$ is used for one-dimensional spectral scanning. The laser source is a tunable laser (TSL 710, SANTEC). The wavelength sweeping range is from $1490 \mathrm{~nm}$ to $1600 \mathrm{~nm}$, which can offer 7.7 degrees of scanning FoV. LiDAR system is based on the FMCW ranging method and spectral scanning approach. The LiDAR system has two interferometer arms. The firstarm is an auxiliary arm that is used to suppress the effects of non-linear optical frequency sweep [28]. The second arm is the measurement arm. By adopting an optical circulator, the transmitting and receiving paths are coaxial. The reflected echo signal from the target is then collected by a fiber collimator (F280APC-1550, Thorlabs) and then captured by a BPD. Here an oscilloscope is used to perform digital data signal acquisition. Then the data is transferred to a personal computer. 3D cloud points can be extracted from the obtained digital signal using short-time Fourier transform (STFT). Detailed description of the working principle of spectral-scanning FMCW LiDAR and its performance can refer to [29, 30]. Here, the FMCW LiDAR system can achieve mm-level ranging accuracy. A zoom fiber collimator (ZC618FC-C, Thorlabs) is used to receive the signal beam in the receiver end. To improve the detectability and recognizability of receiver, a reflective tape with high infrared reflectance is attached around the collimator. Figure. 3 (a) shows a zoomed-in picture of the reflective tape. To distinguish the receiver with other items in the indoor scene, the shape of used marker can be designed into a special shape. As shown in Fig.3 (b), a simple ring is utilized as the marker in the receiver end. The diameter of the circle is around $2 \mathrm{~cm}$, which can be used to distinguish it from other objects with circle shape. The collimator is located at the center position of the ring-like marker.

In the followed subsection 3.2, the detection algorithm based on $3 \mathrm{D}$ could points is introduced and the localization performance is discussed. In subsection 3.3, the experiment of measuring tilt angle of the receiver is conducted and the feasibility of using such method in practical link alignment is also analyzed. Then in subsection 3.4 , a high-data rate transmission link based on our OWC system is demonstrated. (a)

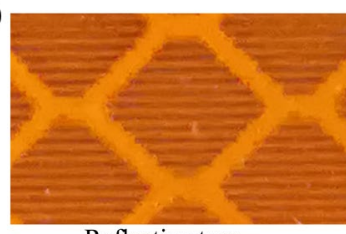

Reflective tape

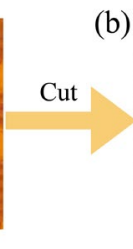

(b)

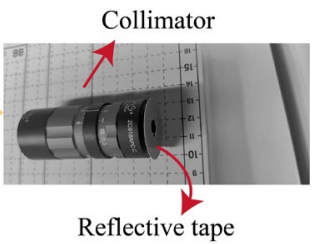

Fig. 3. (a) shows a zoomed-in picture of reflective tape. (b) a ring shape marker is installed on the surface of the receiver collimator.

\subsection{Objection detection and localization based on 3D cloud points}

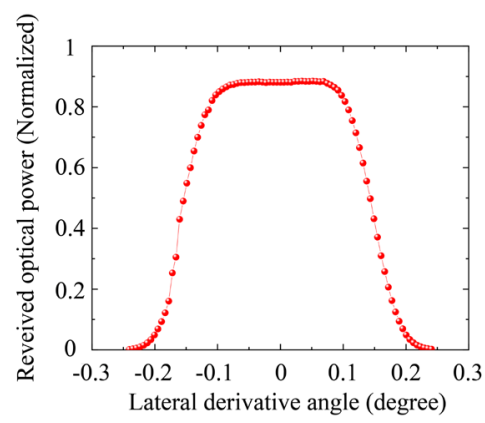

Fig. 4. Experimental results of receive power sensitivity versus the different lateral derivate angles of the incident beam.

In the infrared OWC system, the point-to-point connection method 
leads to the high requirements on the localization accuracy and beam-steering accuracy. Thus, we place the receiver end at $2 \mathrm{~m}$ distance and measure the receive power sensitivity with different beam-steering lateral derivation. As shown in Fig. 4, the full width at half maximum is around 0.15 degrees, which means the localization accuracy of 0.15 degrees is needed. In this section, a simple yet effective object detection pipeline is proposed to detect the circle shape marker within the target scene, locate its center, and approximate its radius, as shown in Fig. 5.

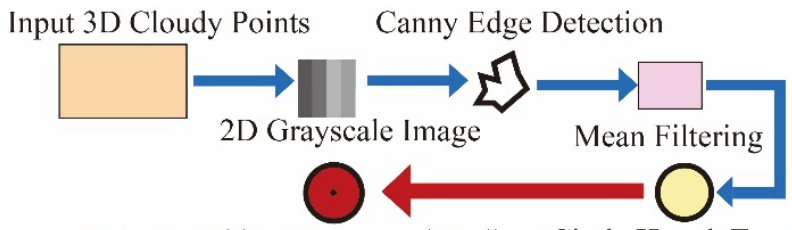

Output: Object Center and Radius Circle Hough Transform

Fig. 5. Proposed framework for object detection of circle shape marker.

Firstly, the 3D point cloud of the target scene with circle shape marker is generated by the aforementioned LiDAR system. Then the matrix of 3D point cloud data is converted to a grayscale image, whose pixel values are proportional to the z-axis values of the 3D data (see Fig. 6. (a)). It can be seen that the grayscale image contains four different objects, i.e., circle, rectangle, triangle, and hexagon. Each of the objects has different pixel values due to their different depths. The next step involves using the well-known Canny edge detector to find out the edges of the four objects. It is a multi-stage algorithm that generally includes 4 steps: using Gaussian filter to remove potential noise that may be misleading from the image;

(a)

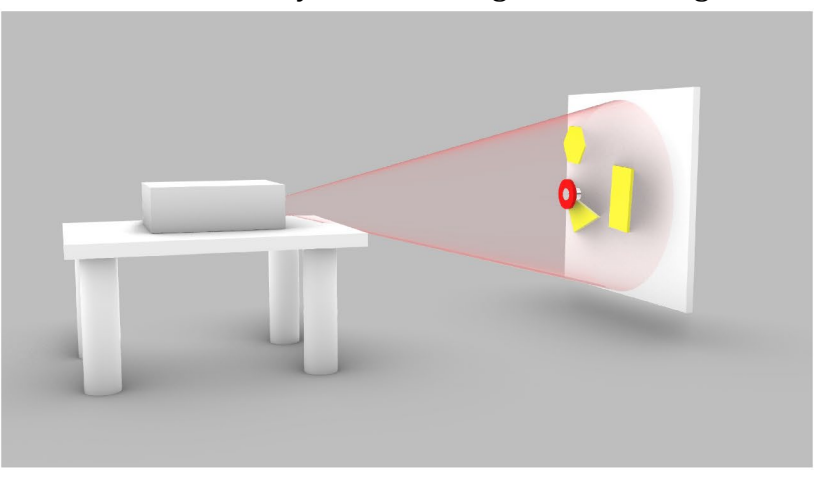

(c)

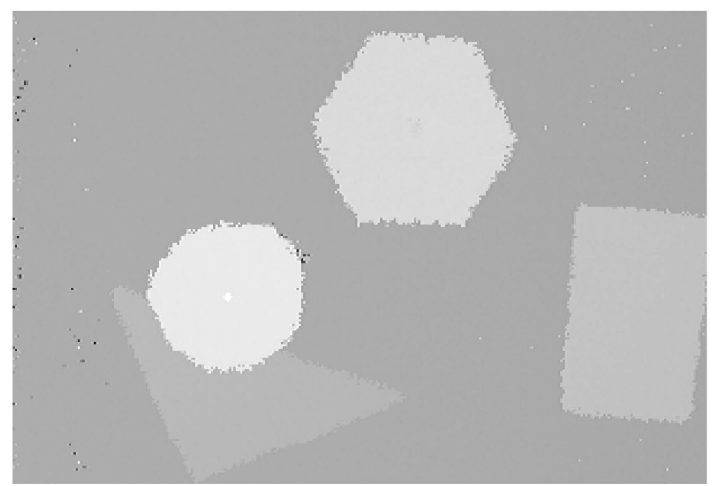

computing the gradients (magnitude and direction) of the image; applying non-maximum suppression to the image to remove spurious response, and finally; combining double threshold and edge tracking to filter out edge pixels with a weak gradient value caused from noise/color variations. After getting the edge detection results, a mean filter with a window size of $3 * 3$ is applied to smooth the image before using circle detection algorithm. The mean filter is a sliding-window spatial filter that replaces the center value in the window with the mean of all values in the window. Finally, circle Hough transform, a specialization of Hough transform, is utilized as a feature extraction method to detect potential circle shapes in the image produced by the above step. Specifically, in our case, since the center coordinates and radius are all unknown, parameter space and accumulator matrix will both be 3D during the process of the circle Hough transform, allowing us to search for circles with an arbitrary radius. Potential circle candidates are obtained by a voting procedure in the Hough parameter space followed by selecting local maxima in an accumulator matrix. As can be seen from the output image in Fig. 6 (d), our proposed pipeline can successfully detect circle shape marker, locate the center, and approximate the radius, with good visual quality.

To further validate the robustness and accuracy of our proposed object detection pipeline, we conduct 10 repeat experiments on the same target scene and compare the detected center position with the ground truth value. The standard angular derivation of 10 times localization measurement is 0.038 degrees, which is relatively small, indicating the superiority and effectiveness of our method. 0.038degree localization accuracy is smaller than the requirement of 0.15 degrees, which shows the feasibility of using such a method to achieve localization.
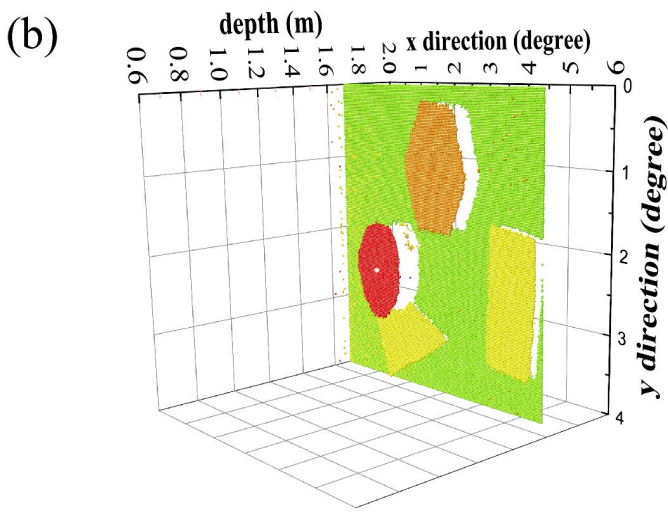

(d)

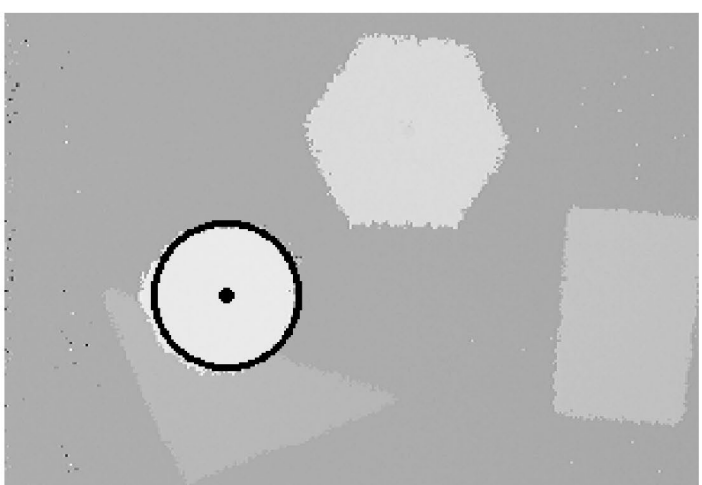

Fig. 6. (a) Experimental scene of detect different objects. (b) 3D imaging results by using FMCW LiDAR system (c) Input: grayscale image converted from 3D point cloud data. (d) Output: detected grayscale image with annotation of center coordinates and radius 


\subsection{Receiver tilt angle measurement}

As discussed above, it is important to acquire the normal direction of receiver surface. In other words, we should know the tilt angle of receiver surface refers to the BSA. It is also important to know whether the utilized BSA is within the FoV of the receiver. Before measuring the tilt angle of the receiver surface, the FoV of the receiver is required. Due to the absence of large effective detect area of high-speed receiver, collimator is utilized in the receiver. To further improve the receiver FoV, a lens with a short focal length is installed before the collimator. The experimental setup is shown in Fig.7 (a). A fiber-pigtailed zoom collimator (ZC618FC-C, Thorlabs) is used in the receiver to optimize the received optical coupling efficiency. The wavelength of laser source is set at $1550 \mathrm{~nm}$. We change the incident angle of signal beam by rotating the transmitter. The results of received optical power with different rotation angles are shown in the Fig.7 (b). We can find that the full width at half maximum angle is 4.6 degrees, which means the full received FoV can reach 9.2 degrees. It also demonstrates that the measuring accuracy of tilt angle of the receiver surface should be better than 4.6 degrees. In the following, we will discuss the performance of tilt angle measurement of the receiver surface by using LiDAR system.

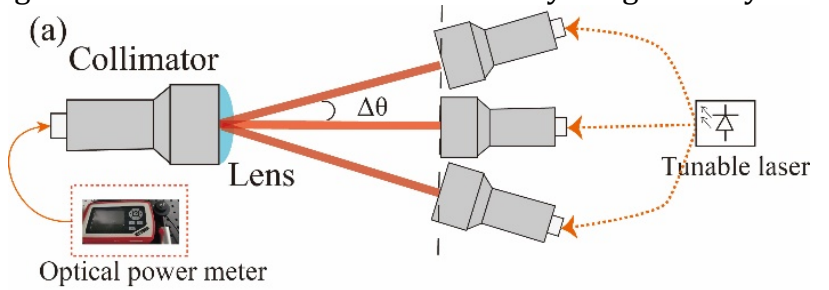

(b)

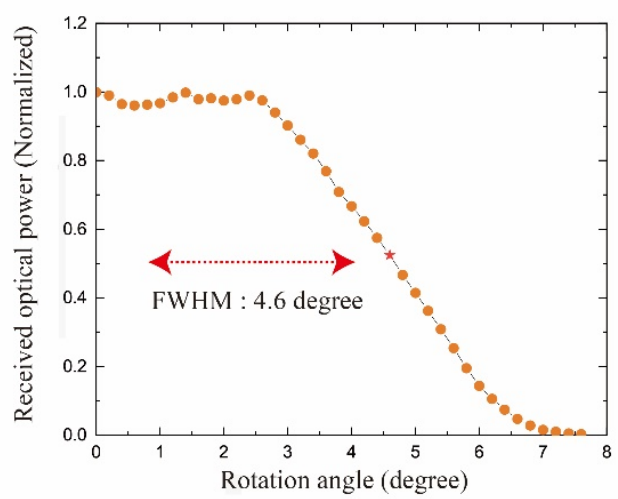

Fig. 7. (a) The experimental setup for the measurement of received optical power coupling efficiency. (b) Received optical power with different rotate angles. FWHM: full width at half maximum.

The experimental setup for tilt angle measurement using LiDAR system is depicted in Fig. 8 (a). The LiDAR system illuminates a $2 \times 2 \mathrm{~cm}^{2}$ square area of the receiver marker. The tunable wavelength range is from $1545 \mathrm{~nm}$ to $1560 \mathrm{~nm}$. The LiDAR system can achieve mm-level ranging accuracy. Based on the obtained 3D cloud points of the receiver surface, we use the general least square method to fit the plane [31]. The widely used least-squares method fits $\mathrm{Z}=\mathrm{a}_{0} \mathrm{X}+\mathrm{a}_{1} \mathrm{Y}+\mathrm{a}_{3}$ to the $3 \mathrm{D}$ cloud points. Then, based on the calculated parameters: $\mathrm{a}_{0}, \mathrm{a}_{1}$ and $\mathrm{a}_{3}$, the tilt angle $\theta$ of the receiver surface refers to the BSA can be calculated. Figure 8(b) shows the derivation between the measured $3 \mathrm{D}$ cloud points and the calculated plane. We can observe that the derivation between the obtained points and the fitting plane is mm-level due to the $\mathrm{mm}$ level ranging accuracy of the LiDAR system. As shown in Fig 8(a), a rotation base is used to change the tilt angle $\theta$ of the receiver and the specific notation of $\theta$ is inserted in Fig. 8(c). The measured angle is close to the standard angle. The standard derivation of twelve measurements is 0.29 degrees. The error bar in the figure shows the 95\% confidence interval of plane fitting results, which gives the estimated interval of high probability containing the true value. The absolute derivation between the minimum value and the maximum value of $95 \%$ confidence interval is smaller than 2 degrees. The rotation angle range is changed from 0 degree to 70 degrees. Both 0.29 degrees standard derivation and 2 degrees $95 \%$ confidence interval range are far less than the receiver FoV. It proves that the angle measurement accuracy by using implemented LiDAR system satisfies the requirement as discussed above.

(a)

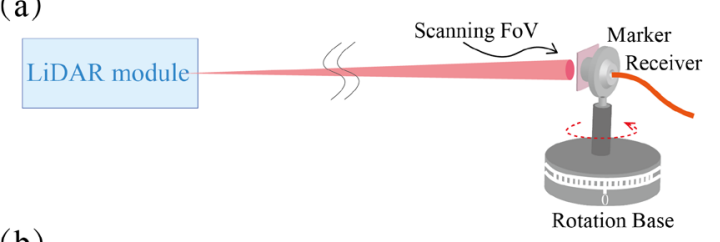

(b)

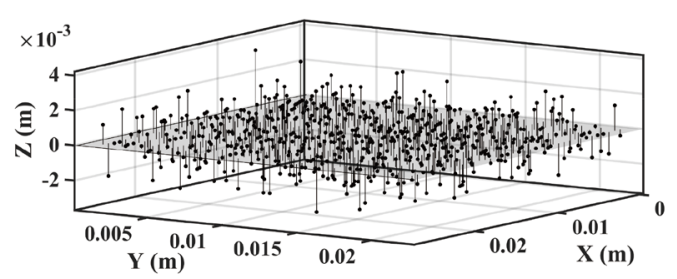

(c)

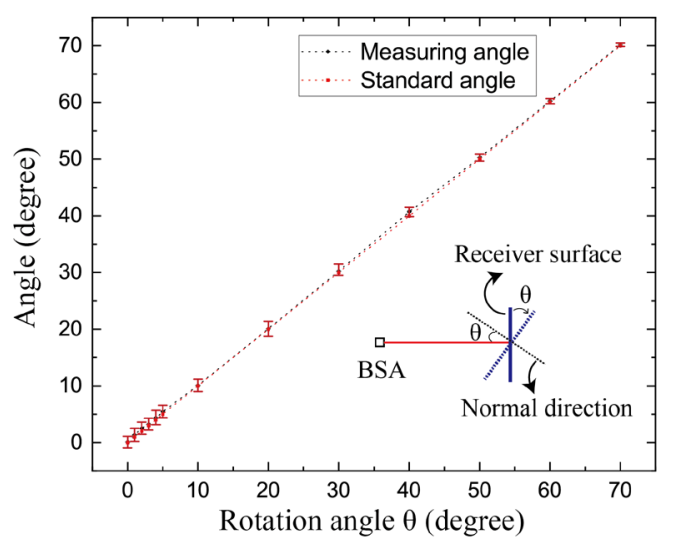

Fig. 8. (a) Experimental setup for rotation angle measurement of receiver surface. (b) Plane fitting results based on the obtained 3D cloud points. (c) The measuring angle results via different rotation angles of receiver.

\subsection{High-capacity data transmission}

In this section, we demonstrate the high data rate transmission link by using the proposed LiDAR integrated OWC system. Figure 2 shows the experimental setup for the proposed high-capacity LiDAR integrated OWC system. To prevent the echo light power which is used for range detection from entering the OWC module, an isolator is used. The OWC system relies on the BSA to steer the signal beam to the user terminals. Based on the beam-steering antenna, the high data transmission data link results are shown in Fig.9. The laser source is a tunable laser (TSL 710, SANTEC). The laser beam is modulated by a Mach-Zehnder modulator (FTM7938EZ, Fujitsu) with non-return-to-zero on-off keying (NRZOOK) modulation format. A pseudorandom bit sequence (PRBS) signal is transmitted into the MZM by a bit error rate tester (BERT, 
MP1900A, Anritsu). In the receiver, an avalanche photodetector (KY-PRM-18G, KY Photonics) is used. The signal from APD is transmitted into the BERT to measure the bit error rate and observe the eye pattern. The free space transmission distance is 3 meters. Here, the diffractive beam-steering method is adopted, and the wavelength-swept range for LiDAR detection is from $1490 \mathrm{~nm}$ to $1600 \mathrm{~nm}$. Thus, the data transmission at different wavelength channels is tested. As demonstrated in Fig. 9(a). The maximum error-free $\left(<10^{-9}\right)$ transmission data rate is $20 \mathrm{Gbps}$. The limitation of transmission data rate is mainly from the bandwidth of APD. The BER performance with different receiver power is presented in Fig. 9(b). Five different wavelength channels have similar data transmission performance. We also measure the received sensitivity at $17 \mathrm{Gbps}$ transmission. When the received power is -22 $\mathrm{dBm}$, the BER is still under the forward error correction (FEC) criterion of $3.8 \times 10^{-3}$. The clear eye diagram can be observed at $1520 \mathrm{~nm}$ and $1600 \mathrm{~nm}$ as shown in Fig.9 (c) and (d).
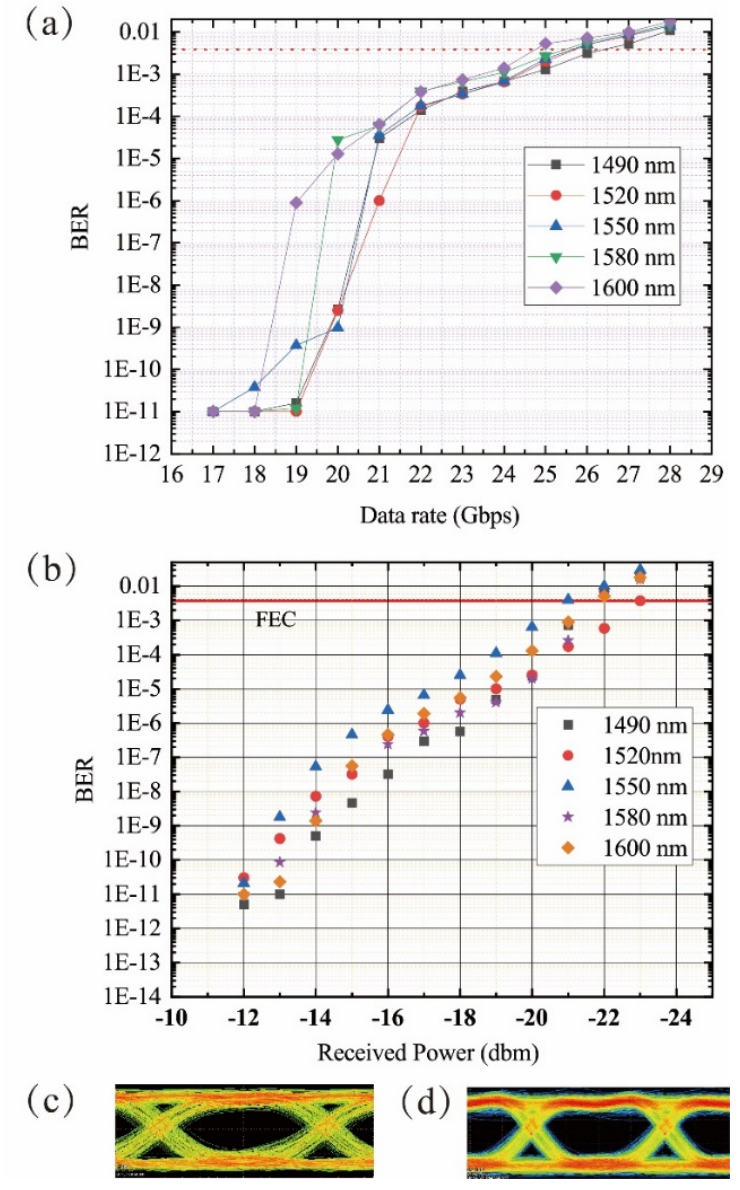

Fig. 9. High-speed data rate transmission. (a) BER performance with different transmission date rate. (b) BER performance versus different received power. (c)(d) The eye diagram of $1520 \mathrm{~nm}$ channel and $1600 \mathrm{~nm}$ channel at $17 \mathrm{Gbps}$ transmission data rate.

\section{Discussion}

In this section, a discussion of potential improvements on object detection method is presented. Alternative beam-steering antennas are also analyzed which can achieve the further improvement of the beam-steering performance and scanning FoV. With the bandwidth limitation of receiver, $20-\mathrm{Gbps}$ error free data transmission is achieved. In subsection 4.3, a discussion of improvement of data transmission capacity is presented. In addition, benefiting from the spectral scanning method, the implemented LiDAR system has reconfigurable 3D imaging ability, which means the localization accuracy is adjustable. This special function is discussed in subsection 4.4 .

\subsection{Objection detection based on 3D cloud points}

In this work, a simple pipeline is developed to detect circle shape marker from 3D point cloud generated by our LiDAR system. Since the pipeline only aims for demonstrating the potential detection and localization ability of the LiDAR system, we only implement simple techniques like Canny edge detection and circle Hough transform. Therefore, the proposed pipeline is limited to the detection of simple geometric shapes. Recently, there are more advanced works that utilize deep learning based method to perform object detection tasks. For example, in [32], researchers propose a model for point cloud based 3D object detection named VoxelNet, which can detect sophisticated objects like cars, pedestrians, and cyclists under autonomous navigation scenario in an end-to-end manner. Although these learning-based methods have better generalization abilities and can work relatively well in more complex scenes, most of them require large datasets for training the model. Under our current experimental equipment, however, generating a single sample already needs a considerable amount of time, making it difficult to employ deep learning methods. In the future, the upgrade of experimental tools may enable us to leverage those more powerful methods.

\subsection{Improvement of BSA}

In this system, we employ a spectrally scnanning method in both beam-steering OWC system and LiDAR system. The advantage of such method is the inertia-free, fast and stable beam-steering. However, owing to the limited dispersion ability, the realizable scanning FoV of such method is limited. The maximum scanning FoV can only reach 20 degrees by using a large line density grating and tunable laser with $100 \mathrm{~nm}$ swept-wavelength range. Many alternate methods can be used such as microelectromechanical systems (MEMS) mirror [33] and optical phased array (OPA) [34] with larger scanning FoV. Besides, a mechanical beam-steering device: galvo-scanner is implemented in the system to achieve a 2D beam-steering ability. It slows down the scanning speed and makes the BSA complicated. Such issues can be solved by employing a VIPA-based 2D disperser. This 2D spectral-scanning method has been introduced in our recent work $[29,30]$.

\subsection{Spectral response}

Due to the bandwidth limitation of APD, we experimentally achieved maximum 20-Gbps free error transmission date rate at 3$\mathrm{m}$ free space distance. The lens and the collimator in the receiver end can be considered as a bandpass filter, which determines the maximum bandwidth of each transmission channel. Thus, we measure the spectral response at different channels. We utilized an amplified spontaneous emission (ASE) light source and an erbium-doped fiber amplifier (EDFA) as a transmitter. The spectral bandwidth in the receiver is captured by an optical spectrum analyzer. As shown in Fig. 10, all the wavelength channels at $1530 \mathrm{~nm}, 1550 \mathrm{~nm}, 1580 \mathrm{~nm}$, and $1600 \mathrm{~nm}$ have 
around $50-\mathrm{GHz}$ bandwidth. It shows that the OWC system can support $50-\mathrm{GHz}$ transmission bandwidth.
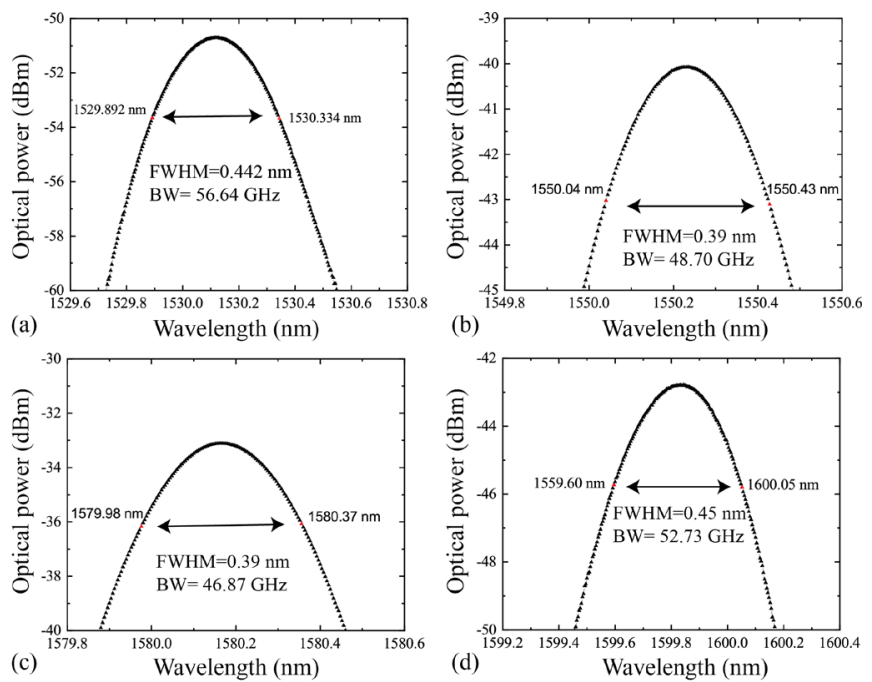

Fig. 10. (a)-(d) Spectrum response at different wavelength channel: 1530 $\mathrm{nm}, 1550 \mathrm{~nm}, 1580 \mathrm{~nm}, 1600 \mathrm{~nm}$.

\subsection{Variable localization accuracy}

The object extraction is based on the 3D cloud points generated by LiDAR system. Thus, the lateral angular resolution of each ranging point has a huge impact on the final localization accuracy. In this subsection, we demonstrate the performance of the LiDAR system on lateral angular resolution.

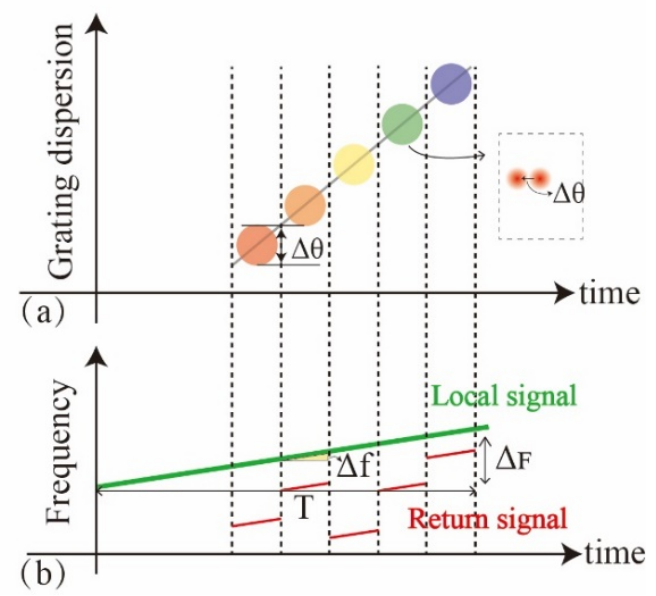

Fig. 11. The ranging principle of spectrally scanned FMCW LiDAR (a) spectral scanning along grating dispersion direction. (b) Ranging principle.

As depicted in Fig. 2, the FMCW measurement is based on a Mach-Zehnder interferometer (MZI) [35]. Figure 11 demonstrates the working principle of the implemented spectrally scanned FMCW LiDAR. The FMCW ranging and beam-steering can be achieved at the same time. During the wavelength sweep process, the output angle of laser beam is changed correspondingly by the grating. As shown in Fig.11, the total tuning bandwidth for a line scanning along $\mathrm{x}$-direction is $\Delta \mathrm{F}$. Then the $\Delta \mathrm{F}$ is divided into multiple sub-bandwidths $\Delta \mathrm{f}$ which are used for each point ranging.
Thus, the number of imaging pixels along $\mathrm{x}$-direction is given by $\mathrm{N}_{\mathrm{x}}=\Delta \mathrm{F} / \Delta \mathrm{f}$. The short-time Fourier transform (STFT) is used to divide each temporal window. Thus, the bandwidth $\Delta \mathrm{f}$ for each ranging point is reconfigurable. As shown in Fig. 11(a), the angular resolution $\Delta \theta$ of each ranging point $\Delta \theta_{\mathrm{m}}$ is mainly determined by the beam angle movement within a detection bandwidth $\Delta \mathrm{f}$. By changing the sub-bandwidth $\Delta \mathrm{f}$, the final angular resolution can be reconfigured. In our localization experiment, the $\Delta \mathrm{f}$ is $0.19 \mathrm{~nm}$. The final angular resolution $\Delta \theta$ is 0.014857 degrees. $\Delta \mathrm{f}$ can be further decreased to improve the angular resolution. Thus the localization accuracy can be improved by sacrificing the ranging resolution. Based on the discussion above, the localization accuracy of our system is variable.

\section{Conclusion}

In this work, we demonstrate a LiDAR integrated infrared OWC system with shared beam-steering device to obtain calibration-free for both localization and tracking. On the one hand, the LiDAR offers the system with high accurate localization ability. We propose a simple circle object detection framework that incorporates Canny edge detection and Hough transform techniques to demonstrate the localization ability of our system. A localization accuracy of 0.038 degrees is achieved. On the other hand, based on the depth information of receiver, the normal direction of the receiver surface can be measured, which offers an excellent solution to the link alignment issues, as discussed in Section 2. The system can achieve an error-free on-off keying (OOK) downlink transmission of $20-\mathrm{Gb} / \mathrm{s}$ in free space at 3$\mathrm{m}$ distance. The maximum data transmission bandwidth can reach $50 \mathrm{GHz}$. The proposed system further demonstrates the feasibility of realizing high-capacity narrow IR optical wireless communication in practical applications.

\section{Acknowledgments.}

The Authors would like to express sincere thanks to the Shenzhen Technology and Innovation Council (WDZC20200820160650001, JCYJ20180507183815699), Overseas Research Cooperation Fund of Tsinghua Shenzhen International Graduate School (HW2020006) and Guangdong Basic and Applied Basic Research Foundation (2021A1515011450).

Disclosures. The authors declare no conflicts of interest.

Data availability. Data underlying the results presented in this paper are not publicly available at this time but may be obtained from the authors upon reasonable request.

\section{Supplemental document. No}

\section{References}

1. J. Gubbi, R. Buyya, S. Marusic, and M. Palaniswami, "Internet of Things (IOT): A vision, architectural elements, and future directions," Future generation computer systems 29, 1645-1660 (2013).

2. H. Elgala, R. Mesleh, and H. Haas, "Indoor optical wireless communication: potential and state-of-the-art," IEEE Communications Magazine 49, 56-62 (2011). 
3. A. Hamza and T. Tripp, "Optical Wireless Communication for the Internet of Things: Advances, Challenges, and Opportunities," (2020).

4. Y. Lu and X. Zheng, "6G: A survey on technologies, scenarios, challenges, and the related issues," Journal of Industrial Information Integration, 100158 (2020).

5. S. ARAI, M. KINOSHITA, and T. YAMAZATO, "Optical Wireless Communication: A Candidate 6G Technology?," IEICE TRANSACTIONS on Fundamentals of Electronics, Communications and Computer Sciences 104, 227-234 (2021).

6. T. Koonen, "Indoor optical wireless systems: technology, trends, and applications," J. Lightwave Technol. 36, 1459-1467 (2017).

7. T. Koonen, J. Oh, K. Mekonnen, and E. Tangdiongga, "Ultra-high capacity indoor optical wireless communication using steered pencil beams," in 2015 International Topical Meeting on Microwave Photonics (MWP), 2015), 1-4.

8. K. Wang, A. Nirmalathas, C. Lim, and E. Skafidas, "High-speed optical wireless communication system for indoor applications," IEEE Photonics Technology Letters 23, 519-521 (2011).

9. A. Gomez, K. Shi, C. Quintana, M. Sato, G. Faulkner, B. C. Thomsen, and D. O'Brien, "Beyond 100-Gb/s indoor wide field-of-view optical wireless communications," IEEE Photonics Technology Letters 27, 367-370 (2014).

10. N. Pham, K. A. Mekonnen, E. Tangdiongga, A. Mefleh, and A. Koonen, "Accurate indoor localization for beam-steered OWC system using optical camera," in 45th European Conference on Optical Communication (ECOC 2019), (IET, 2019), 1-4.

11. A. Gomez, K. Shi, C. Quintana, G. Faulkner, B. C. Thomsen, and D. O'Brien, "A $50 \mathrm{~Gb} / \mathrm{s}$ transparent indoor optical wireless communications link with an integrated localization and tracking system," J. Lightwave Technol. 34, 2510-2517 (2016).

12. W. A. Cahyadi and Y. H. Chung, "Experimental demonstration of indoor uplink near-infrared LED camera communication," Opt. Express 26, 19657-19664 (2018).

13. T. Koonen, K. A. Mekonnen, F. Huijskens, N.-Q. Pham, Z. Cao, and E. Tangdiongga, "Fully passive user localization for beam-steered high-capacity optical wireless communication system," J. Lightwave Technol. 38, 2842-2848 (2020).

$14 . \quad$ C. T. Li, J. C. Cheng, and K. Chen, "Top 10 technologies for indoor positioning on construction sites," Automation in Construction 118, 103309 (2020).

15. A. Rogalski, P. Martyniuk, and M. Kopytko, "Challenges of smallpixel infrared detectors: a review," Reports on Progress in Physics 79, 046501 (2016).

16. A. V. Barve, S. J. Lee, S. K. Noh, and S. Krishna, "Review of current progress in quantum dot infrared photodetectors," Laser \& Photonics Reviews 4, 738-750 (2010).

17. D. V. Hahn, D. M. Brown, N. W. Rolander, J. E. Sluz, and R. Venkat, "Fiber optic bundle array wide field-of-view optical receiver for free space optical communications," Optics Letters 35, 35593561 (2010).

18. G. W. Marsh and J. M. Kahn, "50-Mb/s diffuse infrared free-space link using on-off keying with decision-feedback equalization," IEEE Photonics Technology Letters 6, 1268-1270 (1994).

19. K. Wang, A. Nirmalathas, C. Lim, and E. Skafidas, "Experimental Demonstration of a Full-Duplex Indoor Optical Wireless Communication System," IEEE Photonics Technology Letters 24, 188-190 (2012).

20. D. Droeschel and S. Behnke, "Efficient continuous-time SLAM for 3D lidar-based online mapping," in 2018 IEEE International Conference on Robotics and Automation (ICRA), (IEEE, 2018), 5000-5007.

21. H. Wang, B. Wang, B. Liu, X. Meng, and G. Yang, "Pedestrian recognition and tracking using $3 D$ LiDAR for autonomous vehicle," Robotics and Autonomous Systems 88, 71-78 (2017).

$22 . \quad J$. McCormack, J. Prine, B. Trowbridge, A. C. Rodriguez, and R. Integlia, "2D LIDAR as a distributed interaction tool for virtual and augmented reality video games," in 2015 IEEE Games Entertainment Media Conference (GEM), (IEEE, 2015), 1-5.
23. J. Canny, "A Computational Approach to Edge Detection," IEEE Transactions on Pattern Analysis and Machine Intelligence PAMI8, 679-698 (1986).

24. D. H. Ballard, "Generalizing the Hough transform to detect arbitrary shapes," Pattern recognition 13, 111-122 (1981).

$25 . \quad$ R. O. Duda and P. E. Hart, "Use of the Hough transformation to detect lines and curves in pictures," Communications of the ACM 15, 11-15 (1972).

26. P. V. C. Hough, "Method and means for recognizing complex patterns," (Google Patents, 1962).

27. M. Okano and C. Chong, "Swept Source Lidar: simultaneous FMCW ranging and nonmechanical beam steering with a wideband swept source," Opt. Express 28, 23898-23915 (2020).

28. F. Ito, X. Fan, and Y. Koshikiya, "Long-Range Coherent OFDR With Light Source Phase Noise Compensation," J. Lightwave Technol. 30, 1015-1024 (2012).

$29 . \quad$ Z. Li, Z. Zang, Y. Han, L. Wu, and H. Fu, "Solid-state FMCW LiDAR with two-dimensional spectral scanning using a virtually imaged phased array," Opt. Express 29, 16547-16562 (2021).

$30 . \quad$ Z. Li, Z. Zang, H. Y. Fu, Y. Luo, and Y. Han, "Virtually imaged phased-array-based 2D nonmechanical beam-steering device for FMCW LiDAR," Appl. Opt. 60, 2177-2189 (2021).

31. C. Wang, H. Tanahashi, H. Hirayu, Y. Niwa, and K. Yamamoto, "Comparison of local plane fitting methods for range data," in Proceedings of the 2001 IEEE Computer Society Conference on Computer Vision and Pattern Recognition. CVPR 2001, (IEEE, 2001), I-I.

32. Y. Zhou and O. Tuzel, "Voxelnet: End-to-end learning for point cloud based 3d object detection," in 4490-4499.

33. D. Wang, C. Watkins, and H. Xie, "MEMS mirrors for LiDAR: a review," Micromachines 11, 456 (2020).

34. D. N. Hutchison, J. Sun, J. K. Doylend, R. Kumar, J. Heck, W. Kim, C. T. Phare, A. Feshali, and H. Rong, "High-resolution aliasing-free optical beam steering," Optica 3, 887-890 (2016).

35. B. Behroozpour, P. A. Sandborn, M. C. Wu, and B. E. Boser, "Lidar system architectures and circuits," IEEE Communications Magazine 55, 135-142 (2017). 